\title{
Supplementary Information: Thermal Evolution of Internal Strain in Doped PbTe
}

\author{
James P. Male ${ }^{1+}$, Riley Hanus ${ }^{2 *+}$, G. Jeffrey Snyder ${ }^{1 *}$, and Raphaël P. Hermann ${ }^{2}$ \\ ${ }^{1}$ Materials Science and Engineering, Northwestern University, Evanston, IL 60208, USA. \\ ${ }^{2}$ Materials Science and Technology Division, Oak Ridge National Laboratory, Oak Ridge, TN 37831, USA. \\ *Corresponding author: hanusriley@gmail.com, jeff.snyder@northwestern.edu \\ +J.P.M. and R.H. contributed equally to this work
}

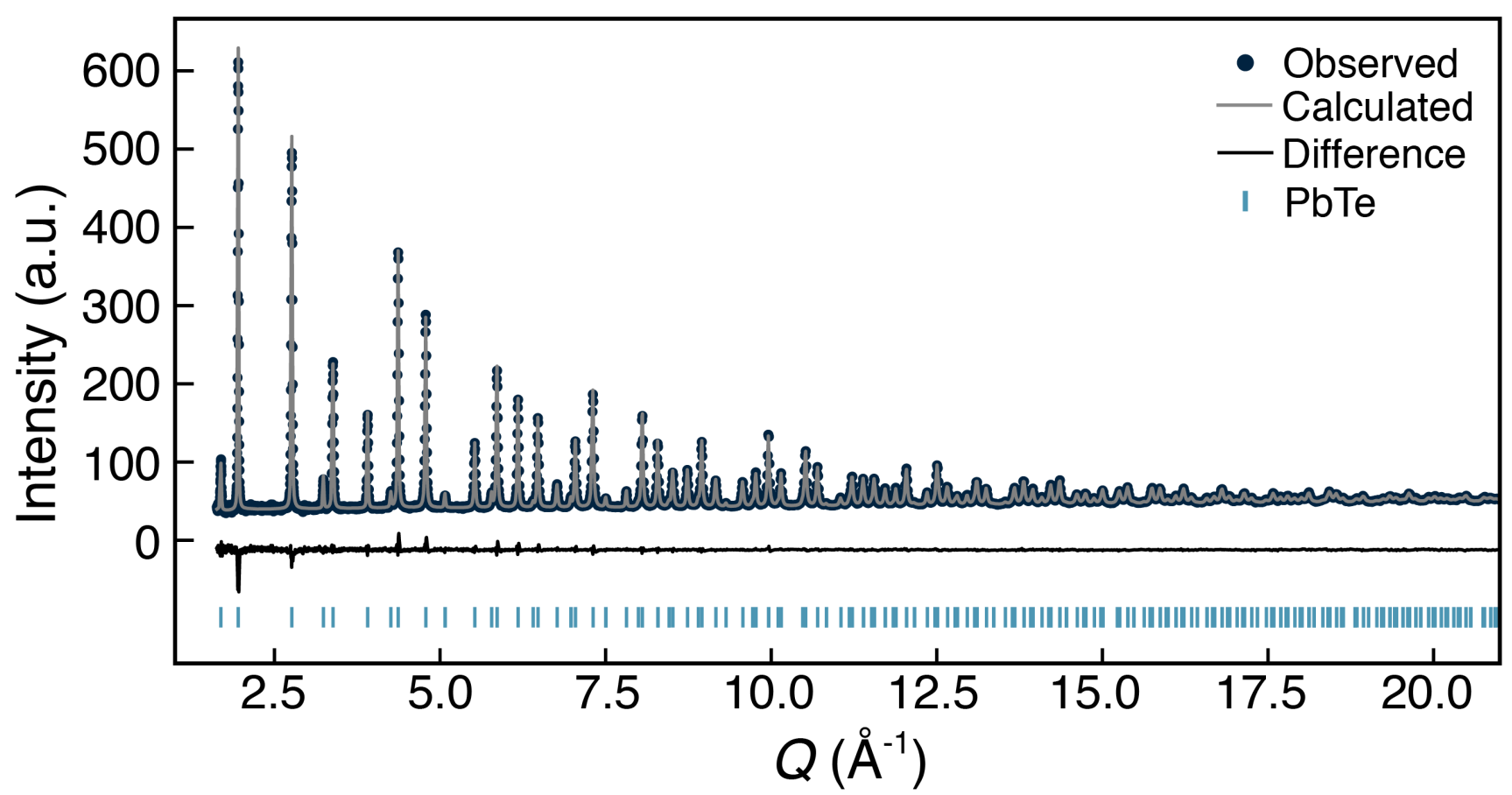

Figure 1. Neutron diffraction pattern taken on $\mathrm{Pb}_{0.98} \mathrm{Na}_{0.02}$ Te powder ball milled for 15 minutes showing full $Q$ range used for Rietveld Refinement in GSAS-II. Discernible peaks from the rock salt PbTe structure $(F m \overline{3} m)$ are refined up to $Q$ near $20 \AA^{-1}$. $w R$ for this fit is 1.51 , and refinements at higher temperature tend to have improved statistics. 

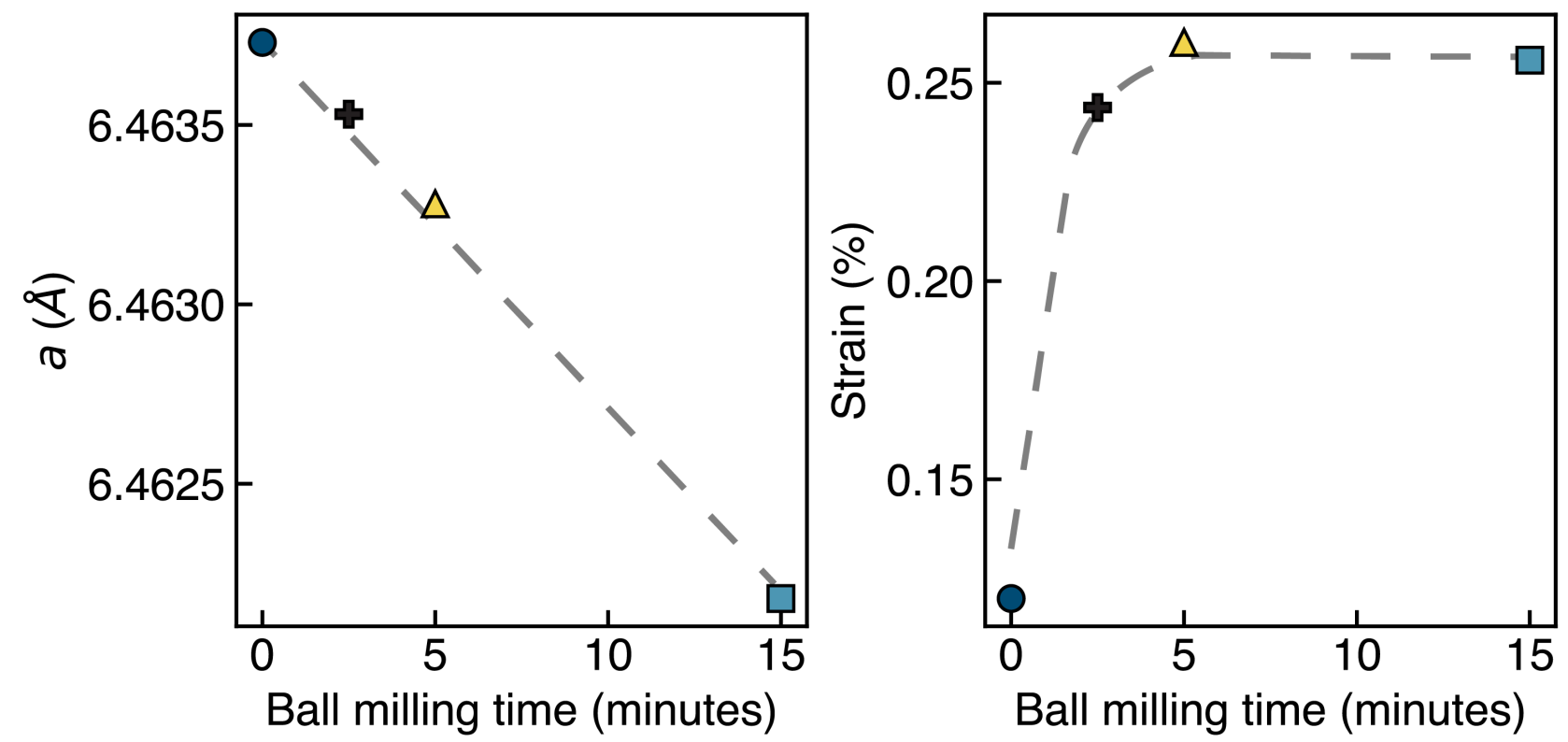

Figure 2. (a) Room temperature lattice parameter (a) and (b) strain in undoped PbTe powders high energy ball milled for 0, $2.5,5$, or 15 minutes. The $a$ decreases linearly with ball milling time, while strain determined by Rietveld refinement saturates between 2.5 and 5 minutes of ball milling. Dotted lines are drawn as guides to the eye.
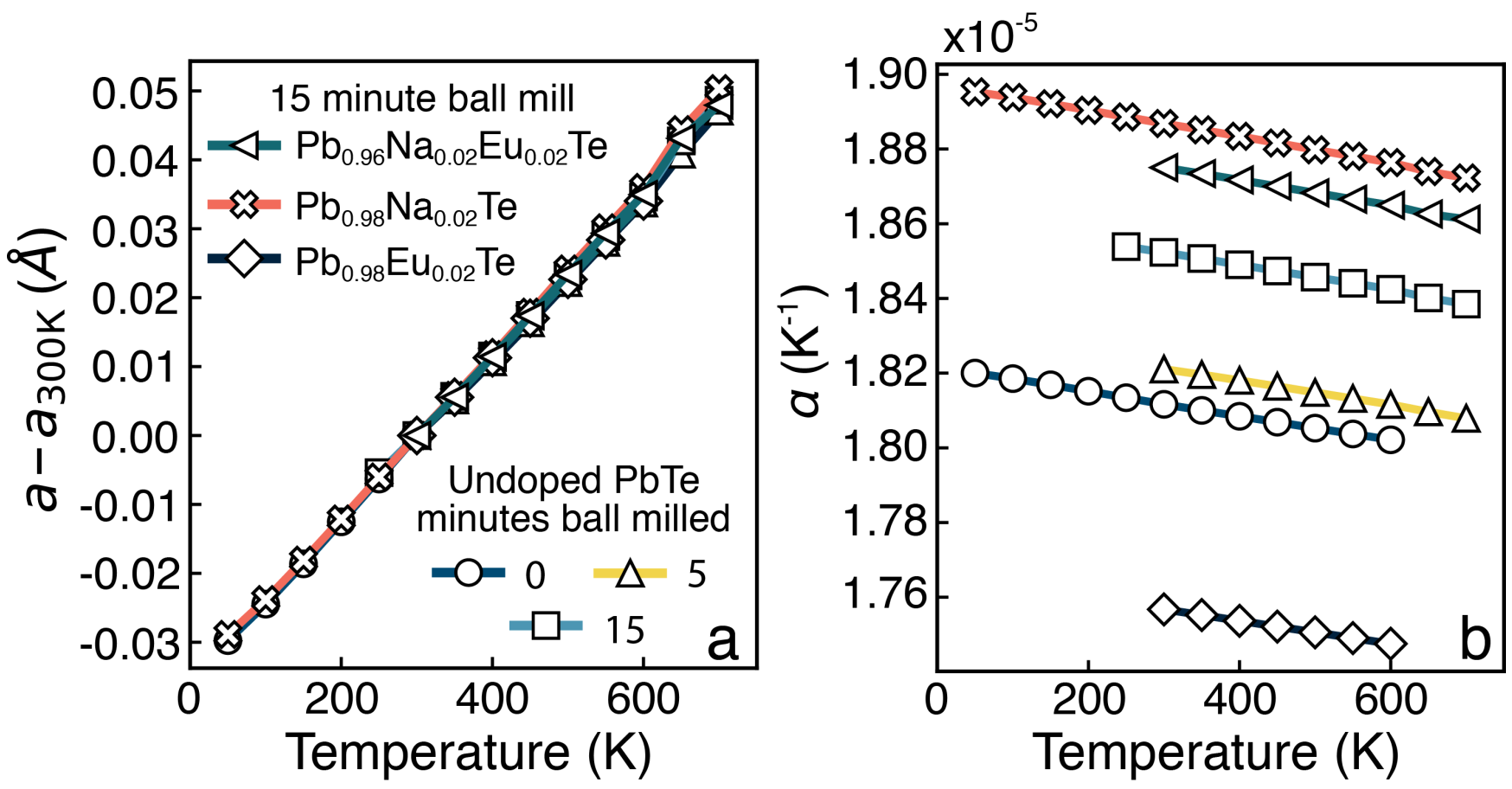

Figure 3. (a) Temperature dependent change in lattice parameter $(a)$ relative to $a$ at $300 \mathrm{~K}\left(a_{300 \mathrm{~K}}\right)$. (b) First order coefficient of thermal expansion $(\alpha)$ and its temperature dependence. Each sample has $\alpha$ within $10 \%$ of the undoped, unmilled sample. 

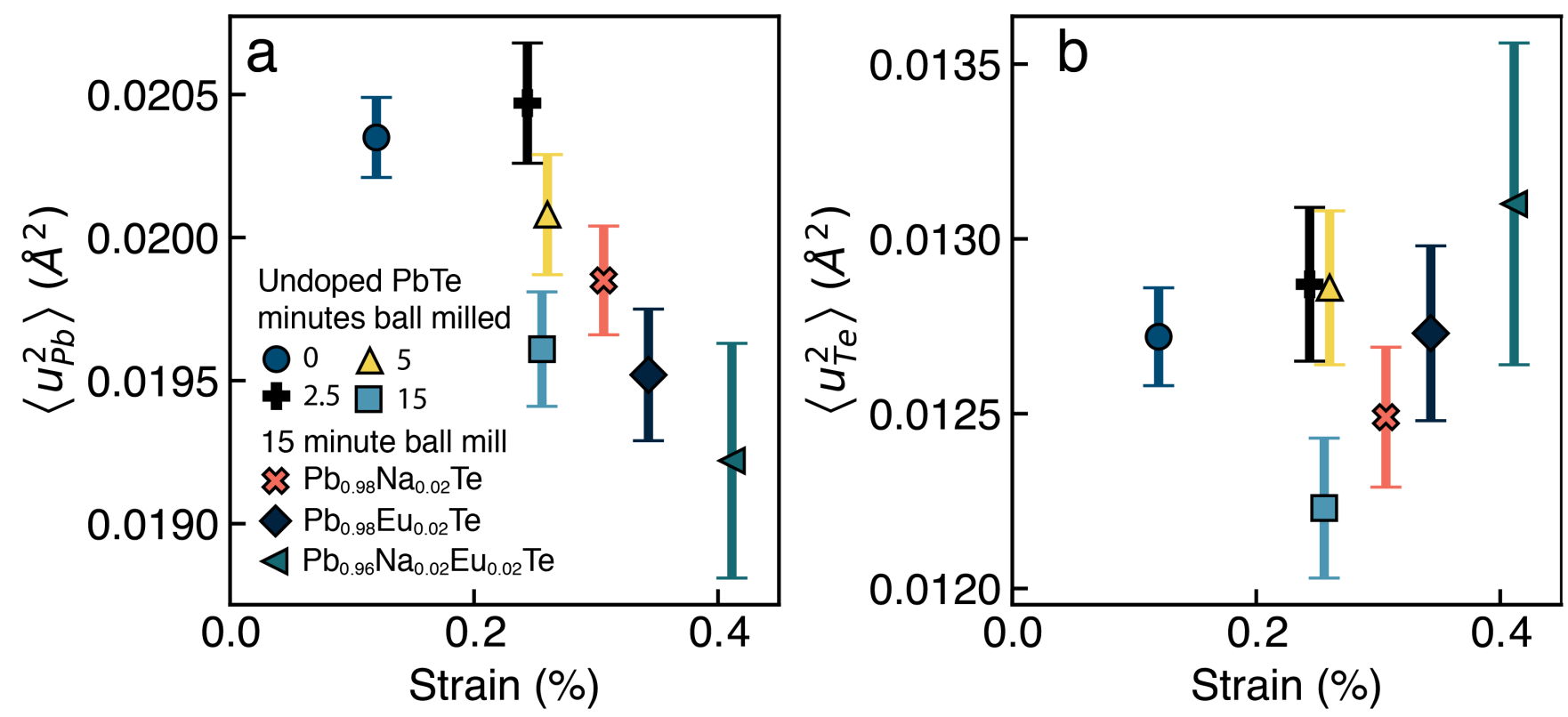

Figure 4. (a) Room temperature isotropic thermal displacement parameters for (a) $\mathrm{Pb}\left(\left\langle u_{P b}^{2}\right\rangle\right)$ and (b) $\mathrm{Te}\left(\left\langle u_{T e}^{2}\right\rangle\right)$ in undoped $\mathrm{PbTe}$ ball milled for 0-15 minutes and $\mathrm{Pb}$ doped with $\mathrm{Na}$ and/or Eu ball milled for 15 minutes versus strain determined by Rietveld refinement.

\section{Debye temperatures and frequency weighting of different techniques}

The Debye phonon dispersion approximation is a simple tool for calculating thermodynamic parameters for a material which may, in reality, have a complicated phonon band structure. Experimental $\theta_{\mathrm{D}}$ values are calculated from measured cutoff frequencies $\left(\omega_{D}\right)$ for a Debye distribution - not the true cutoff frequency of a solid's phonon dispersion. To find $\theta_{\mathrm{D}}$, one measures crystal properties (commonly: speed of sound, heat capacity, or Debye-Waller factors) and relates them to a Debye distribution using simplified models. In a perfect Debye distribution, each measured property gives identical temperatureindependent $\theta_{\mathrm{D}}$. In practice, each technique is weighted differently by phonon frequency i.e., each measurement probes a different $n$ 'th moment, $\overline{\omega^{n}}$, of the phonon density of states $(G(\omega))$, defined as:

$$
\overline{\omega^{n}} \sim \int_{0}^{\omega_{D}} \omega^{n} G(\omega) d \omega
$$

Higher $n$ moments will more heavily weight high frequency phonons and may better capture changes to optical branches in the phonon dispersion. Measurements that probe a lower $n$ will be more sensitive to changes in near-zero frequency phonons and acoustic branches ${ }^{1}$. Therefore, different experiments for finding $\theta_{\mathrm{D}}$ are making fundamentally different measurements, and close agreement between the $\theta_{\mathrm{D}}$ found by different techniques should not be compared expecting close agreement.

Meticulous explanations distinguishing the moments probed by different measurement techniques are available elsewhere ${ }^{2 \mid 5}$. In Table 1, we provide a brief summary of experimental $\theta_{\mathrm{D}, \mathrm{PbTe}}, \theta_{\mathrm{D}, \mathrm{Pb}}$, and $\theta_{\mathrm{D}, \mathrm{Te}}$ values measured in other works. We also note where the PbTe-based samples were intentionally prepared to have a high or low degree of internal strain from dislocations ("strained" or "unstrained", respectively), and where the samples were in pellet or powder form. Reduced $\theta_{\mathrm{D}}$ values have been measured on strained $\mathrm{PbTe}$ samples in pellet form using speed of sound and low temperature heat capacity measurements, which is what one might expect if lattice softening is present and elastic constants are reduced ${ }^{6-8}$. Low temperature heat capacity probes the $n=-3$ moment of the $\operatorname{DOS}^{2}$. Speed of sound gives $\theta_{\mathrm{D}}$ using an averaging scheme that mirrors $n=-3$, but actually measures the near 0 -frequency phonons. Note that, while speed of sound measurements probe low phonon frequencies, anharmonic softening can lead to departures from the heat capacity $\theta_{\mathrm{D}}$ at high temperatures and any systematic deviation is dependent on the material and measurement system in question 5 . High temperature Debye-Waller analyses like our study probe the $n=-2$ moment of the DOS, and low temperature Debye-Waller measurements are weighted towards $n=-149$. A $\theta_{\mathrm{D}}$ approximation using Extended X-ray absorption fine structure analysis (EXAFS) is more complex. In non-Bravais lattice materials, the use of the correlated Debye model is not recommend ${ }^{10}$. Other useful moments of the density of states can be analyzed by the phonon DOS zero point energy $(n=1)$, and high temperature heat capacity $(n=2 \sqrt{415}$. A measurement's sensitivity to low frequency phonons decreases at higher $n$. Therefore, measurements like low temperature heat capacity or 
Table 1. Collection of Debye temperatures $\left(\theta_{\mathrm{D}}\right)$ for $\mathrm{Pb}, \mathrm{Te}$, and $\mathrm{PbTe}$ found from room temperature speed of sound measurements, low temperature heat capacity, atomic displacement parameters (ADPs) from neutron or X-ray diffraction (XRD) - used in a Debye-Waller analysis - and from an Extended X-ray absorption fine structure (EXAFS) Debye-Waller type analysis. Instances are noted where the PbTe was intentionally strained during synthesis. Low temperature specifies measurements near $0 \mathrm{~K}$. The moment $n$ of the density of states probed by each method is indicated. Note that $\theta_{\mathrm{D}}$ is generally different between each measurement method, but comparable between different measurements from the same technique. A softening of elastic constants (reduced $\theta_{\mathrm{D}}$ ) is apparent from speed of sound and heat capacity measurements, but not an ADP analysis. Uncertainty noted where it is provided in the original works.

\begin{tabular}{|c|c|c|c|c|c|c|c|}
\hline Moment $(n)$ & Method & "Strained"? & Temperature & Form & $\theta_{\mathrm{D}, \mathrm{Te}}$ & $\theta_{\mathrm{D}, \mathrm{Pb}}$ & $\theta_{\mathrm{D}, \mathrm{PbTe}}$ \\
\hline-3 & Speed of sound & Yes & High & Pellet & - & - & 163 \\
\hline-3 & Speed of sound 11 & Yes & High & Pellet & - & - & 164 \\
\hline-3 & Speed of sound & Yes & High & Pellet & - & - & 159 \\
\hline-3 & Speed of sound & No & High & Pellet & - & - & 172 \\
\hline-3 & Speed of sound & No & High & Pellet & - & - & $175(3)$ \\
\hline-3 & Speed of sound & No & High & Pellet & - & - & 136 \\
\hline-3 & Heat capacity & Yes & Low & Pellet & - & - & 135 \\
\hline-3 & Heat capacity & No & Low & Pellet & - & - & 150 \\
\hline-3 & Heat capacity 15 & No & Low & Pellet & - & - & 178 \\
\hline-3 & Heat capacity 16 & No & Low & Pellet & - & - & 168 \\
\hline-2 & Neutron powder diffraction ADP ${ }^{1}$ & No & High & Powder & $163(1)$ & $102(1)$ & 133(1) \\
\hline-2 & Neutron powder diffraction ADPs & Yes & High & Powder & $165(2)$ & 104(1) & $134(2)$ \\
\hline-2 & Neutron single crystal diffraction ADPs 17 & No & High & Pellet & $162(3)$ & 101(2) & $132(3)$ \\
\hline-2 & Neutron powder diffraction ADPs 17 & No & High & Powder & $157(11)$ & $123(9)$ & $140(14)$ \\
\hline-2 & Nuclear inelastic scattering ADP\$18 & No & Low & Powder & $170(5)$ & - & - \\
\hline-2 & Synchrotron powder XRD ADPs 19 & No & High & Powder & $151(2)$ & $100(1)$ & $125(3)$ \\
\hline-2 & Neutron powder diffraction ADPs 20 & No & High & Powder & - & 104(1) & - \\
\hline Complex & EXAFS 21 & No & High & Powder & 120 & 116 & - \\
\hline Complex & EXAFS 22 & No & High & Powder & 109 & 121 & - \\
\hline
\end{tabular}

i: This study unstrained, undoped $\mathrm{PbTe}$.

ii: This study, $\mathrm{Pb}_{0.98} \mathrm{Na}_{0.02} \mathrm{Te}$ ball milled 15 minutes

speed of sound would be best for capturing the effects of long-range strain from dislocations, which may soften low frequency phonons without significant changes at higher frequencies. While more experiments are needed, the lack of softening from neutron powder diffraction atomic displacements suggests that strain primarily softens the lowest frequency phonons in PbTe or that the character of strain is different between powders and pellets. 
Table 2. Full refinement statistics and crystallographic information determined by Rietveld refinement in the GSAS-II software

\begin{tabular}{|c|c|c|c|c|c|c|c|c|c|}
\hline Composition & Min Milled & $\mathrm{T}(\mathrm{K})$ & $\bar{a}$ & $U_{i s o_{P b}}$ & $U_{\text {iso }_{T e}}$ & $\alpha\left(\mathrm{x} 10^{-5} \mathrm{~K}^{-1}\right)$ & $\mathrm{wR}(\%)$ & GOF & T Cycle \\
\hline $\mathrm{PbTe}$ & 0 & 50 & $6.433982(15)$ & $0.00337(3)$ & $0.00229(4)$ & 1.82 & 2.82 & 3.05 & Heating \\
\hline $\mathrm{PbTe}$ & 0 & 100 & $6.439268(16)$ & $0.00647(5)$ & $0.00389(5)$ & 1.819 & 1.96 & 2.5 & Heating \\
\hline $\mathrm{PbTe}$ & 0 & 150 & $6.445108(18)$ & $0.00979(7)$ & $0.00582(7)$ & 1.817 & 1.95 & 2.34 & Heating \\
\hline $\mathrm{PbTe}$ & 0 & 200 & $6.451215(20)$ & $0.01315(9)$ & $0.00807(9)$ & 1.815 & 1.76 & 2.1 & Heating \\
\hline $\mathrm{PbTe}$ & 0 & 250 & $6.457419(25)$ & $0.01669(12)$ & $0.01024(13)$ & 1.813 & 2.14 & 2.21 & Heating \\
\hline $\mathrm{PbTe}$ & 0 & 300 & $6.463733(26)$ & $0.02035(14)$ & $0.01272(14)$ & 1.812 & 1.8 & 1.96 & Heating \\
\hline $\mathrm{PbTe}$ & 0 & 350 & $6.469492(32)$ & $0.02383(19)$ & $0.01519(19)$ & 1.81 & 1.77 & 1.52 & Heating \\
\hline $\mathrm{PbTe}$ & 0 & 400 & $6.475146(36)$ & $0.02715(24)$ & $0.01717(23)$ & 1.808 & 2 & 1.52 & Heating \\
\hline $\mathrm{PbTe}$ & 0 & 450 & $6.480830(40)$ & $0.03035(26)$ & $0.0199(26)$ & 1.807 & 1.99 & 1.5 & Heating \\
\hline $\mathrm{PbTe}$ & 0 & 500 & $6.486554(43)$ & $0.03379(31)$ & $0.02211(30)$ & 1.805 & 1.92 & 1.48 & Heating \\
\hline $\mathrm{PbTe}$ & 0 & 550 & $6.492170(44)$ & $0.03695(32)$ & $0.02488(32)$ & 1.804 & 1.92 & 1.41 & Heating \\
\hline $\mathrm{PbTe}$ & 0 & 600 & $6.497794(49)$ & $0.04004(38)$ & $0.02727(38)$ & 1.802 & 1.88 & 1.47 & Heating \\
\hline $\mathrm{PbTe}$ & 0 & 519 & $6.490936(77)$ & $0.03794(61)$ & $0.02381(57)$ & - & 3.12 & 1.08 & Cooling \\
\hline $\mathrm{PbTe}$ & 0 & $472(25)$ & $6.485421(55)$ & $0.03311(39)$ & $0.02179(38)$ & - & 2.48 & 1.12 & Cooling \\
\hline $\mathrm{PbTe}$ & 0 & $422(25)$ & $6.479000(43)$ & $0.0294(28)$ & $0.01922(28)$ & - & 2 & 1.23 & Cooling \\
\hline $\mathrm{PbTe}$ & 0 & $372(25)$ & $6.473183(34)$ & $0.02623(21)$ & $0.01701(21)$ & - & 1.67 & 1.28 & Cooling \\
\hline $\mathrm{PbTe}$ & 2.5 & $300(25)$ & $6.463527(47)$ & $0.02047(21)$ & $0.01287(22)$ & - & 5.66 & 1.25 & Heating \\
\hline $\mathrm{PbTe}$ & 5 & 300 & $6.463279(50)$ & $0.02008(21)$ & $0.01286(22)$ & 1.821 & 1.69 & 1.35 & Heating \\
\hline $\mathrm{PbTe}$ & 5 & 350 & $6.468433(61)$ & $0.02319(28)$ & $0.0148(29)$ & 1.82 & 1.9 & 1.2 & Heating \\
\hline $\mathrm{PbTe}$ & 5 & 400 & $6.473956(67)$ & $0.02625(33)$ & $0.01729(34)$ & 1.818 & 1.89 & 1.22 & Heating \\
\hline $\mathrm{PbTe}$ & 5 & 450 & $6.479633(68)$ & $0.02968(38)$ & $0.01892(38)$ & 1.816 & 1.96 & 1.18 & Heating \\
\hline $\mathrm{PbTe}$ & 5 & 500 & $6.485463(69)$ & $0.03244(42)$ & $0.02167(42)$ & 1.815 & 1.91 & 1.21 & Heating \\
\hline $\mathrm{PbTe}$ & 5 & 550 & $6.491301(70)$ & $0.03624(47)$ & $0.02391(47)$ & 1.813 & 2.05 & 1.19 & Heating \\
\hline $\mathrm{PbTe}$ & 5 & 600 & $6.497012(72)$ & $0.04002(54)$ & $0.0257(52)$ & 1.812 & 2.06 & 1.2 & Heating \\
\hline $\mathrm{PbTe}$ & 5 & 650 & $6.504104(75)$ & $0.04285(57)$ & $0.02956(58)$ & 1.81 & 2.03 & 1.23 & Heating \\
\hline $\mathrm{PbTe}$ & 5 & 700 & $6.510339(73)$ & $0.04762(64)$ & $0.03237(63)$ & 1.808 & 2.15 & 1.22 & Heating \\
\hline $\mathrm{PbTe}$ & 5 & 613 & $6.506934(149)$ & $0.04554(126)$ & $0.03024(124)$ & - & 3.52 & 1.06 & Cooling \\
\hline $\mathrm{PbTe}$ & 5 & $573(25)$ & $6.496281(122)$ & $0.03908(88)$ & $0.02704(90)$ & - & 3.83 & 1.06 & Cooling \\
\hline $\mathrm{PbTe}$ & 5 & $522(25)$ & $6.490167(88)$ & $0.0357(65)$ & $0.02406(65)$ & - & 3.12 & 1.09 & Cooling \\
\hline $\mathrm{PbTe}$ & 5 & $472(25)$ & $6.484300(68)$ & $0.03261(48)$ & $0.02115(47)$ & - & 2.45 & 1.1 & Cooling \\
\hline $\mathrm{PbTe}$ & 5 & $422(25)$ & $6.478366(48)$ & $0.02883(32)$ & $0.01882(32)$ & - & 1.99 & 1.14 & Cooling \\
\hline $\mathrm{PbTe}$ & 5 & $372(25)$ & $6.472946(40)$ & $0.02573(25)$ & $0.0169(25)$ & - & 1.85 & 1.2 & Cooling \\
\hline $\mathrm{PbTe}$ & 15 & $250(25)$ & $6.456798(42)$ & $0.01672(16)$ & $0.01017(17)$ & 1.854 & 1.6 & 1.29 & Heating \\
\hline $\mathrm{PbTe}$ & 15 & 300 & $6.462180(47)$ & $0.01961(20)$ & $0.01223(20)$ & 1.852 & 1.62 & 1.27 & Heating \\
\hline $\mathrm{PbTe}$ & 15 & 350 & $6.467860(53)$ & $0.02238(24)$ & $0.01441(24)$ & 1.851 & 1.61 & 1.26 & Heating \\
\hline $\mathrm{PbTe}$ & 15 & 400 & $6.473723(58)$ & $0.02589(29)$ & $0.01604(29)$ & 1.849 & 1.68 & 1.26 & Heating \\
\hline $\mathrm{PbTe}$ & 15 & 450 & $6.479602(57)$ & $0.02898(31)$ & $0.01885(31)$ & 1.847 & 1.67 & 1.23 & Heating \\
\hline $\mathrm{PbTe}$ & 15 & 500 & $6.485345(58)$ & $0.03184(34)$ & $0.02128(34)$ & 1.846 & 1.57 & 1.24 & Heating \\
\hline $\mathrm{PbTe}$ & 15 & 550 & $6.491047(58)$ & $0.03552(39)$ & $0.02348(39)$ & 1.844 & 1.72 & 1.22 & Heating \\
\hline $\mathrm{PbTe}$ & 15 & 600 & $6.496765(61)$ & $0.03909(43)$ & $0.02587(43)$ & 1.842 & 1.9 & 1.27 & Heating \\
\hline $\mathrm{PbTe}$ & 15 & 650 & $6.504921(60)$ & $0.04349(47)$ & $0.02979(47)$ & 1.84 & 1.83 & 1.3 & Heating \\
\hline $\mathrm{PbTe}$ & 15 & 700 & $6.510819(61)$ & $0.04729(52)$ & $0.0323(52)$ & 1.838 & 2.04 & 1.31 & Heating \\
\hline $\mathrm{PbTe}$ & 15 & 622 & $6.507828(95)$ & $0.04619(80)$ & $0.03146(79)$ & - & 2.48 & 1.09 & Cooling \\
\hline $\mathrm{Pb}_{0.98} \mathrm{Eu}_{0.02} \mathrm{Te}$ & 15 & $300(25)$ & $6.466538(61)$ & $0.01952(23)$ & $0.01273(25)$ & 1.757 & 5.13 & 1.36 & Heating \\
\hline $\mathrm{Pb}_{0.98} \mathrm{Eu}_{0.02} \mathrm{Te}$ & 15 & 350 & $6.472092(90)$ & $0.0231(41)$ & $0.0142(41)$ & 1.755 & 1.34 & 1.17 & Heating \\
\hline $\mathrm{Pb}_{0.98} \mathrm{Eu}_{0.02} \mathrm{Te}$ & 15 & 400 & $6.477824(95)$ & $0.02528(42)$ & $0.01708(45)$ & 1.754 & 1.58 & 1.15 & Heating \\
\hline $\mathrm{Pb}_{0.98} \mathrm{Eu}_{0.02} \mathrm{Te}$ & 15 & 450 & $6.483549(98)$ & $0.02796(47)$ & $0.01932(51)$ & 1.752 & 1.29 & 1.16 & Heating \\
\hline $\mathrm{Pb}_{0.98} \mathrm{Eu}_{0.02} \mathrm{Te}$ & 15 & 500 & $6.489187(97)$ & $0.03163(55)$ & $0.02201(59)$ & 1.751 & 1.32 & 1.16 & Heating \\
\hline $\mathrm{Pb}_{0.98} \mathrm{Eu}_{0.02} \mathrm{Te}$ & 15 & 550 & $6.494918(99)$ & $0.03521(62)$ & $0.02372(63)$ & 1.749 & 1.27 & 1.15 & Heating \\
\hline $\mathrm{Pb}_{0.98} \mathrm{Eu}_{0.02} \mathrm{Te}$ & 15 & 600 & $6.500565(103)$ & $0.03922(75)$ & $0.0255(74)$ & 1.748 & 1.65 & 1.12 & Heating \\
\hline $\mathrm{Pb}_{0.98} \mathrm{Na}_{0.02} \mathrm{Te}$ & 15 & 50 & $6.431687(28)$ & $0.00345(4)$ & $0.00265(6)$ & 1.895 & 1.51 & 1.58 & Heating \\
\hline $\mathrm{Pb}_{0.98} \mathrm{Na}_{0.02} \mathrm{Te}$ & 15 & 100 & $6.436746(31)$ & $0.00633(6)$ & $0.00404(8)$ & 1.894 & 1.46 & 1.54 & Heating \\
\hline $\mathrm{Pb}_{0.98} \mathrm{Na}_{0.02} \mathrm{Te}$ & 15 & 150 & $6.442475(36)$ & $0.00952(9)$ & $0.00581(10)$ & 1.892 & 1.34 & 1.49 & Heating \\
\hline $\mathrm{Pb}_{0.98} \mathrm{Na}_{0.02} \mathrm{Te}$ & 15 & 200 & $6.448444(38)$ & $0.01288(12)$ & $0.00791(13)$ & 1.89 & 1.67 & 1.35 & Heating \\
\hline $\mathrm{Pb}_{0.98} \mathrm{Na}_{0.02} \mathrm{Te}$ & 15 & 250 & $6.454530(42)$ & $0.01642(15)$ & $0.01019(16)$ & 1.889 & 1.27 & 1.3 & Heating \\
\hline $\mathrm{Pb}_{0.98} \mathrm{Na}_{0.02} \mathrm{Te}$ & 15 & 300 & $6.460557(48)$ & $0.01985(19)$ & $0.01249(20)$ & 1.887 & 1.39 & 1.3 & Heating \\
\hline
\end{tabular}


Table 2 continued from previous page

\begin{tabular}{|c|c|c|c|c|c|c|c|c|c|}
\hline Composition & Min Milled & $\mathrm{T}(\mathrm{K})$ & $a$ & $U_{\text {iso }_{P b}}$ & $U_{\text {iso }_{T e}}$ & $\alpha\left(\mathrm{x} 10^{-5} \mathrm{~K}^{-1}\right)$ & wR (\%) & GOF & T Cycle \\
\hline $\mathrm{Pb}_{0.98} \mathrm{Na}_{0.02} \mathrm{Te}$ & 15 & 350 & $6.466384(60)$ & $0.02324(27)$ & $0.01461(28)$ & 1.885 & 1.48 & 1.19 & Heating \\
\hline $\mathrm{Pb}_{0.98} \mathrm{Na}_{0.02} \mathrm{Te}$ & 15 & 400 & $6.472293(64)$ & $0.02574(31)$ & $0.0166(31)$ & 1.883 & 1.48 & 1.17 & Heating \\
\hline $\mathrm{Pb}_{0.98} \mathrm{Na}_{0.02} \mathrm{Te}$ & 15 & 450 & $6.478449(64)$ & $0.02901(34)$ & $0.01942(35)$ & 1.882 & 1.61 & 1.24 & Heating \\
\hline $\mathrm{Pb}_{0.98} \mathrm{Na}_{0.02} \mathrm{Te}$ & 15 & 500 & $6.484719(57)$ & $0.03299(38)$ & $0.02152(38)$ & 1.88 & 1.62 & 1.22 & Heating \\
\hline $\mathrm{Pb}_{0.98} \mathrm{Na}_{0.02} \mathrm{Te}$ & 15 & 550 & $6.490691(58)$ & $0.03607(42)$ & $0.02376(42)$ & 1.878 & 1.74 & 1.25 & Heating \\
\hline $\mathrm{Pb}_{0.98} \mathrm{Na}_{0.02} \mathrm{Te}$ & 15 & 600 & $6.496541(58)$ & $0.03898(44)$ & $0.02625(44)$ & 1.876 & 1.76 & 1.23 & Heating \\
\hline $\mathrm{Pb}_{0.98} \mathrm{Na}_{0.02} \mathrm{Te}$ & 15 & 650 & $6.504927(60)$ & $0.04483(51)$ & $0.02991(50)$ & 1.874 & 1.7 & 1.23 & Heating \\
\hline $\mathrm{Pb}_{0.98} \mathrm{Na}_{0.02} \mathrm{Te}$ & 15 & 700 & $6.510879(64)$ & $0.04821(56)$ & $0.03303(56)$ & 1.872 & 1.93 & 1.26 & Heating \\
\hline $\mathrm{Pb}_{0.98} \mathrm{Na}_{0.02} \mathrm{Te}$ & 15 & 656 & $6.509827(191)$ & $0.04867(178)$ & $0.03047(173)$ & - & 7.31 & 1.04 & Cooling \\
\hline $\mathrm{Pb}_{0.98} \mathrm{Na}_{0.02} \mathrm{Te}$ & 15 & $622(25)$ & $6.507282(90)$ & $0.0467(78)$ & $0.03165(78)$ & - & 2.65 & 1.06 & Cooling \\
\hline $\mathrm{Pb}_{0.98} \mathrm{Na}_{0.02} \mathrm{Te}$ & 15 & $572(25)$ & $6.501878(74)$ & $0.04369(62)$ & $0.02925(61)$ & - & 2.15 & 1.11 & Cooling \\
\hline $\mathrm{Pb}_{0.98} \mathrm{Na}_{0.02} \mathrm{Te}$ & 15 & $522(25)$ & $6.495688(60)$ & $0.04031(50)$ & $0.02598(48)$ & - & 1.95 & 1.14 & Cooling \\
\hline $\mathrm{Pb}_{0.98} \mathrm{Na}_{0.02} \mathrm{Te}$ & 15 & $472(25)$ & $6.488785(45)$ & $0.03517(32)$ & $0.0237(32)$ & - & 1.87 & 1.18 & Cooling \\
\hline $\mathrm{Pb}_{0.98} \mathrm{Na}_{0.02} \mathrm{Te}$ & 15 & $432(25)$ & $6.481133(63)$ & $0.0303(38)$ & $0.02046(38)$ & - & 1.54 & 1.24 & Cooling \\
\hline $\mathrm{Pb}_{0.98} \mathrm{Na}_{0.02} \mathrm{Te}$ & 15 & $378(25)$ & $6.471440(49)$ & $0.02513(32)$ & $0.01601(32)$ & - & 1.81 & 1.14 & Cooling \\
\hline $\mathrm{Pb}_{0.96} \mathrm{Na}_{0.02} \mathrm{Eu}_{0.02} \mathrm{Te}$ & 15 & $300(25)$ & $6.463869(113)$ & $0.01922(41)$ & $0.0131(46)$ & 1.875 & 1.35 & 1.14 & Heating \\
\hline $\mathrm{Pb}_{0.96} \mathrm{Na}_{0.02} \mathrm{Eu}_{0.02} \mathrm{Te}$ & 15 & 350 & $6.469409(104)$ & $0.02267(44)$ & $0.01429(46)$ & 1.873 & 6.43 & 1.15 & Heating \\
\hline $\mathrm{Pb}_{0.96} \mathrm{Na}_{0.02} \mathrm{Eu}_{0.02} \mathrm{Te}$ & 15 & 400 & $6.475279(103)$ & $0.02562(48)$ & $0.01599(50)$ & 1.872 & 1.49 & 1.13 & Heating \\
\hline $\mathrm{Pb}_{0.96} \mathrm{Na}_{0.02} \mathrm{Eu}_{0.02} \mathrm{Te}$ & 15 & 450 & $6.481236(96)$ & $0.02878(52)$ & $0.01884(54)$ & 1.87 & 1.48 & 1.14 & Heating \\
\hline $\mathrm{Pb}_{0.96} \mathrm{Na}_{0.02} \mathrm{Eu}_{0.02} \mathrm{Te}$ & 15 & 500 & $6.487262(94)$ & $0.03244(59)$ & $0.02132(59)$ & 1.868 & 1.61 & 1.19 & Heating \\
\hline $\mathrm{Pb}_{0.96} \mathrm{Na}_{0.02} \mathrm{Eu}_{0.02} \mathrm{Te}$ & 15 & 550 & $6.493239(91)$ & $0.03633(69)$ & $0.02253(67)$ & 1.867 & 1.94 & 1.14 & Heating \\
\hline $\mathrm{Pb}_{0.96} \mathrm{Na}_{0.02} \mathrm{Eu}_{0.02} \mathrm{Te}$ & 15 & 600 & $6.498830(91)$ & $0.03827(65)$ & $0.02671(68)$ & 1.865 & 1.32 & 1.14 & Heating \\
\hline $\mathrm{Pb}_{0.96} \mathrm{Na}_{0.02} \mathrm{Eu}_{0.02} \mathrm{Te}$ & 15 & 650 & $6.507002(96)$ & $0.04484(82)$ & $0.02939(84)$ & 1.863 & 2.04 & 1.16 & Heating \\
\hline $\mathrm{Pb}_{0.96} \mathrm{Na}_{0.02} \mathrm{Eu}_{0.02} \mathrm{Te}$ & 15 & 700 & $6.511804(98)$ & $0.04962(99)$ & $0.031(94)$ & 1.861 & 1.95 & 1.2 & Heating \\
\hline $\mathrm{Pb}_{0.96} \mathrm{Na}_{0.02} \mathrm{Eu}_{0.02} \mathrm{Te}$ & 15 & 666 & $6.510520(257)$ & $0.04721(232)$ & $0.03035(230)$ & - & 4.05 & 1.07 & Cooling \\
\hline $\mathrm{Pb}_{0.96} \mathrm{Na}_{0.02} \mathrm{Eu}_{0.02} \mathrm{Te}$ & 15 & $622(25)$ & $6.507626(179)$ & $0.04618(162)$ & $0.02956(158)$ & - & 2.81 & 1.04 & Cooling \\
\hline $\mathrm{Pb}_{0.96} \mathrm{Na}_{0.02} \mathrm{Eu}_{0.02} \mathrm{Te}$ & 15 & $576(25)$ & $6.495425(269)$ & $0.03683(171)$ & $0.027(188)$ & - & 4.01 & 1.05 & Cooling \\
\hline $\mathrm{Pb}_{0.96} \mathrm{Na}_{0.02} \mathrm{Eu}_{0.02} \mathrm{Te}$ & 15 & $522(25)$ & $6.489328(182)$ & $0.03502(136)$ & $0.02254(136)$ & - & 3.37 & 1.05 & Cooling \\
\hline $\mathrm{Pb}_{0.96} \mathrm{Na}_{0.02} \mathrm{Eu}_{0.02} \mathrm{Te}$ & 15 & $472(25)$ & $6.483498(139)$ & $0.03084(91)$ & $0.02113(95)$ & - & 2.6 & 1.06 & Cooling \\
\hline $\mathrm{Pb}_{0.96} \mathrm{Na}_{0.02} \mathrm{Eu}_{0.02} \mathrm{Te}$ & 15 & $422(25)$ & $6.477474(100)$ & $0.02794(61)$ & $0.0194(65)$ & - & 2.44 & 1.08 & Cooling \\
\hline $\mathrm{Pb}_{0.96} \mathrm{Na}_{0.02} \mathrm{Eu}_{0.02} \mathrm{Te}$ & 15 & $372(25)$ & $6.471786(78)$ & $0.02515(49)$ & $0.01638(49)$ & - & 1.65 & 1.09 & Cooling \\
\hline
\end{tabular}

Table 3. Full refinement statistics and strain information determined by Rietveld refinement in the GSAS-II software

\begin{tabular}{lllllll}
\hline Composition & Min Milled & $\mathrm{T}(\mathrm{K})$ & $\mathrm{S} 400$ & $\mathrm{~S} 220$ & $\mu$ strain & T Cycle \\
\hline $\mathrm{PbTe}$ & 0 & 50 & $378.4(18.1)$ & $564.8(18.6)$ & 955.5 & Heating \\
$\mathrm{PbTe}$ & 0 & 100 & $497.6(21.2)$ & $696.7(20.8)$ & 1079.5 & Heating \\
$\mathrm{PbTe}$ & 0 & 150 & $544.2(25.1)$ & $734.5(23.8)$ & 1120.6 & Heating \\
$\mathrm{PbTe}$ & 0 & 200 & $533.4(26.3)$ & $787.1(25.2)$ & 1137.1 & Heating \\
$\mathrm{PbTe}$ & 0 & 250 & $660.4(34.8)$ & $772.1(32.3)$ & 1197.2 & Heating \\
$\mathrm{PbTe}$ & 0 & 300 & $596.1(34.0)$ & $859.2(32.0)$ & 1199.4 & Heating \\
$\mathrm{PbTe}$ & 0 & 350 & $556.2(41.2)$ & $935.3(38.3)$ & 1209.4 & Heating \\
$\mathrm{PbTe}$ & 0 & 400 & $615.5(47.6)$ & $872.4(42.6)$ & 1217.9 & Heating \\
$\mathrm{PbTe}$ & 0 & 450 & $647.1(52.7)$ & $785.7(44.1)$ & 1204.3 & Heating \\
$\mathrm{PbTe}$ & 0 & 500 & $532.0(51.3)$ & $812.2(45.5)$ & 1158.4 & Heating \\
$\mathrm{PbTe}$ & 0 & 550 & $541.0(53.8)$ & $746.4(45.0)$ & 1140.0 & Heating \\
$\mathrm{PbTe}$ & 0 & 600 & $460.9(56.5)$ & $785.9(49.7)$ & 1114.9 & Heating \\
$\mathrm{PbTe}$ & 0 & $519(25)$ & $599.7(95.4)$ & $585.1(72.1)$ & 1107.7 & Cooling \\
$\mathrm{PbTe}$ & 0 & $472(25)$ & $491.5(63.2)$ & $729.5(53.3)$ & 1104.8 & Cooling \\
$\mathrm{PbTe}$ & 0 & $422(25)$ & $553.6(53.4)$ & $823.0(48.4)$ & 1170.8 & Cooling \\
$\mathrm{PbTe}$ & 0 & $372(25)$ & $524.5(41.3)$ & $746.4(36.1)$ & 1124.7 & Cooling \\
$\mathrm{PbTe}$ & 2.5 & 300 & $1929.5(112.3)$ & $4272.3(116.5)$ & 2437.5 & Heating \\
$\mathrm{PbTe}$ & 5 & 300 & $2241.4(128.3)$ & $4809.5(131.3)$ & 2601.7 & Heating \\
$\mathrm{PbTe}$ & 5 & 350 & $2278.6(159.1)$ & $4736.6(155.6)$ & 2602.1 & Heating \\
$\mathrm{PbTe}$ & 5 & 400 & $2125.8(168.9)$ & $4699.4(166.3)$ & 2565.5 & Heating \\
$\mathrm{PbTe}$ & 5 & 450 & $1899.8(160.6)$ & $4018.5(153.5)$ & 2396.9 & Heating \\
\hline
\end{tabular}


Table 3 continued from previous page

\begin{tabular}{|c|c|c|c|c|c|c|}
\hline Composition & Min Milled & $\mathrm{T}(\mathrm{K})$ & $\mathrm{S} 400$ & S220 & $\mu$ strain & T Cycle \\
\hline $\mathrm{PbTe}$ & 5 & 500 & $1498.7(146.6)$ & $3014.7(137.1)$ & 2100.6 & Heating \\
\hline $\mathrm{PbTe}$ & 5 & 550 & $1170.1(130.0)$ & $2396.6(120.8)$ & 1887.8 & Heating \\
\hline $\mathrm{PbTe}$ & 5 & 600 & $943.1(119.7)$ & 1870.7(108.6) & 1665.3 & Heating \\
\hline $\mathrm{PbTe}$ & 5 & 650 & $680.0(105.8)$ & $1384.4(98.1)$ & 1428.2 & Heating \\
\hline $\mathrm{PbTe}$ & 5 & 700 & $476.8(86.9)$ & $816.7(73.0)$ & 1139.8 & Heating \\
\hline $\mathrm{PbTe}$ & 5 & $613(25)$ & $575.8(110.8)$ & $685.1(89.4)$ & 1131.9 & Cooling \\
\hline $\mathrm{PbTe}$ & 5 & $573(25)$ & $567.7(83.7)$ & $696.3(67.7)$ & 1133.1 & Cooling \\
\hline $\mathrm{PbTe}$ & 5 & $522(25)$ & $590.5(154.3)$ & $910.1(125.5)$ & 1113.7 & Cooling \\
\hline $\mathrm{PbTe}$ & 5 & $472(25)$ & $468.3(170.1)$ & $764.7(143.0)$ & 1227.2 & Cooling \\
\hline $\mathrm{PbTe}$ & 5 & $422(25)$ & $512.5(57.5)$ & $724.2(49.0)$ & 1111.6 & Cooling \\
\hline $\mathrm{PbTe}$ & 5 & $372(25)$ & $523.7(48.4)$ & $636.0(41.5)$ & 1080.8 & Cooling \\
\hline $\mathrm{PbTe}$ & 15 & 250 & $2399.8(123.8)$ & 4331.1(120.4) & 2513.3 & Heating \\
\hline $\mathrm{PbTe}$ & 15 & 300 & $2581.3(143.3)$ & $4639.8(135.9)$ & 2556.8 & Heating \\
\hline $\mathrm{PbTe}$ & 15 & 350 & $2686.4(162.5)$ & 4495.1(142.8) & 2653.4 & Heating \\
\hline $\mathrm{PbTe}$ & 15 & 400 & $2165.1(142.8)$ & $3526.9(122.1)$ & 2657.8 & Heating \\
\hline $\mathrm{PbTe}$ & 15 & 450 & $1725.6(128.6)$ & 2717.2(111.5) & 2372.9 & Heating \\
\hline $\mathrm{PbTe}$ & 15 & 500 & $1381.3(115.9)$ & 2151.3(99.6) & 2102.7 & Heating \\
\hline $\mathrm{PbTe}$ & 15 & 550 & $1086.5(104.2)$ & $1477.3(82.3)$ & 1879.1 & Heating \\
\hline $\mathrm{PbTe}$ & 15 & 600 & 2256.4(109.9) & 4293.8(108.4) & 1611.9 & Heating \\
\hline $\mathrm{PbTe}$ & 15 & 650 & $769.5(88.1)$ & $939.0(69.6)$ & 1324.6 & Heating \\
\hline $\mathrm{PbTe}$ & 15 & 700 & $712.2(255.5)$ & $763.1(182.3)$ & 1134.7 & Heating \\
\hline $\mathrm{PbTe}$ & 15 & $622(25)$ & $440.5(106.7)$ & $680.7(85.5)$ & 1064.4 & Cooling \\
\hline $\mathrm{Pb}_{0.98} \mathrm{Eu}_{0.02} \mathrm{Te}$ & 15 & 300 & $4484.0(221.6)$ & $7537.3(202.3)$ & 3430.5 & Heating \\
\hline $\mathrm{Pb}_{0.98} \mathrm{Eu}_{0.02} \mathrm{Te}$ & 15 & 350 & $4708.4(339.1)$ & 7668.2(297.4) & 3490.9 & Heating \\
\hline $\mathrm{Pb}_{0.98} \mathrm{Eu}_{0.02} \mathrm{Te}$ & 15 & 400 & $4419.8(345.2)$ & $7486.5(305.1)$ & 3425.0 & Heating \\
\hline $\mathrm{Pb}_{0.98} \mathrm{Eu}_{0.02} \mathrm{Te}$ & 15 & 450 & $4013.5(340.0)$ & $6135.6(287.3)$ & 3179.9 & Heating \\
\hline $\mathrm{Pb}_{0.98} \mathrm{Eu}_{0.02} \mathrm{Te}$ & 15 & 500 & $2891.5(288.3)$ & $4879.7(248.6)$ & 2777.2 & Heating \\
\hline $\mathrm{Pb}_{0.98} \mathrm{Eu}_{0.02} \mathrm{Te}$ & 15 & 550 & $2771.8(283.8)$ & $3982.7(231.3)$ & 2609.4 & Heating \\
\hline $\mathrm{Pb}_{0.98} \mathrm{Eu}_{0.02} \mathrm{Te}$ & 15 & 600 & $2286.9(271.6)$ & $3252.3(213.7)$ & 2368.0 & Heating \\
\hline $\mathrm{Pb}_{0.98} \mathrm{Na}_{0.02} \mathrm{Te}$ & 15 & 50 & $2132.2(73.3)$ & $5722.0(90.5)$ & 2698.8 & Heating \\
\hline $\mathrm{Pb}_{0.98} \mathrm{Na}_{0.02} \mathrm{Te}$ & 15 & 100 & $3006.3(92.2)$ & $6461.6(103.8)$ & 2989.9 & Heating \\
\hline $\mathrm{Pb}_{0.98} \mathrm{Na}_{0.02} \mathrm{Te}$ & 15 & 150 & $3354.7(112.7)$ & $6740.8(120.5)$ & 3100.3 & Heating \\
\hline $\mathrm{Pb}_{0.98} \mathrm{Na}_{0.02} \mathrm{Te}$ & 15 & 200 & $3459.7(122.7)$ & $6842.4(123.9)$ & 3139.4 & Heating \\
\hline $\mathrm{Pb}_{0.98} \mathrm{Na}_{0.02} \mathrm{Te}$ & 15 & 250 & $3115.9(129.5)$ & $6624.7(130.2)$ & 3050.6 & Heating \\
\hline $\mathrm{Pb}_{0.98} \mathrm{Na}_{0.02} \mathrm{Te}$ & 15 & 300 & $3231.4(147.9)$ & $6515.8(146.8)$ & 3063.1 & Heating \\
\hline $\mathrm{Pb}_{0.98} \mathrm{Na}_{0.02} \mathrm{Te}$ & 15 & 350 & $3542.9(197.9)$ & $6725.0(185.3)$ & 3156.3 & Heating \\
\hline $\mathrm{Pb}_{0.98} \mathrm{Na}_{0.02} \mathrm{Te}$ & 15 & 400 & $3368.0(204.7)$ & $6207.8(186.7)$ & 3056.9 & Heating \\
\hline $\mathrm{Pb}_{0.98} \mathrm{Na}_{0.02} \mathrm{Te}$ & 15 & 450 & $2442.4(169.1)$ & $3678.5(146.1)$ & 2467.0 & Heating \\
\hline $\mathrm{Pb}_{0.98} \mathrm{Na}_{0.02} \mathrm{Te}$ & 15 & 500 & $1346.6(112.1)$ & 1903.4(94.1) & 1805.4 & Heating \\
\hline $\mathrm{Pb}_{0.98} \mathrm{Na}_{0.02} \mathrm{Te}$ & 15 & 550 & 1017.1(98.4) & $1459.8(82.6)$ & 1578.2 & Heating \\
\hline $\mathrm{Pb}_{0.98} \mathrm{Na}_{0.02} \mathrm{Te}$ & 15 & 600 & $744.3(84.2)$ & $1179.9(71.2)$ & 1388.3 & Heating \\
\hline $\mathrm{Pb}_{0.98} \mathrm{Na}_{0.02} \mathrm{Te}$ & 15 & 650 & $568.4(77.5)$ & $938.8(65.2)$ & 1229.9 & Heating \\
\hline $\mathrm{Pb}_{0.98} \mathrm{Na}_{0.02} \mathrm{Te}$ & 15 & 700 & $540.7(80.2)$ & $756.9(62.8)$ & 1150.4 & Heating \\
\hline $\mathrm{Pb}_{0.98} \mathrm{Na}_{0.02} \mathrm{Te}$ & 15 & $656(25)$ & $446.7(217.8)$ & $836.5(189.6)$ & 1131.4 & Cooling \\
\hline $\mathrm{Pb}_{0.98} \mathrm{Na}_{0.02} \mathrm{Te}$ & 15 & $622(25)$ & $496.3(71.3)$ & $901.7(62.1)$ & 1131.6 & Cooling \\
\hline $\mathrm{Pb}_{0.98} \mathrm{Na}_{0.02} \mathrm{Te}$ & 15 & $572(25)$ & $612.4(63.7)$ & $530.8(50.5)$ & 1159.5 & Cooling \\
\hline $\mathrm{Pb}_{0.98} \mathrm{Na}_{0.02} \mathrm{Te}$ & 15 & $522(25)$ & 1318.3(116.6) & 1331.6(86.9) & 1177.0 & Cooling \\
\hline $\mathrm{Pb}_{0.98} \mathrm{Na}_{0.02} \mathrm{Te}$ & 15 & $472(25)$ & $681.0(60.3)$ & $771.1(48.0)$ & 1218.6 & Cooling \\
\hline $\mathrm{Pb}_{0.98} \mathrm{Na}_{0.02} \mathrm{Te}$ & 15 & $432(25)$ & 513.1(88.5) & $826.3(73.0)$ & 1649.0 & Cooling \\
\hline $\mathrm{Pb}_{0.98} \mathrm{Na}_{0.02} \mathrm{Te}$ & 15 & $378(25)$ & $490.2(105.5)$ & $780.2(88.4)$ & 1086.4 & Cooling \\
\hline $\mathrm{Pb}_{0.96} \mathrm{Na}_{0.02} \mathrm{Eu}_{0.02} \mathrm{Te}$ & 15 & 300 & $5549.5(472.3)$ & $12068.4(462.2)$ & 4114.4 & Heating \\
\hline $\mathrm{Pb}_{0.96} \mathrm{Na}_{0.02} \mathrm{Eu}_{0.02} \mathrm{Te}$ & 15 & 350 & $6445.5(467.2)$ & $12047.9(423.9)$ & 4242.2 & Heating \\
\hline $\mathrm{Pb}_{0.96} \mathrm{Na}_{0.02} \mathrm{Eu}_{0.02} \mathrm{Te}$ & 15 & 400 & $5484.7(424.3)$ & $10806.6(388.0)$ & 3981.3 & Heating \\
\hline $\mathrm{Pb}_{0.96} \mathrm{Na}_{0.02} \mathrm{Eu}_{0.02} \mathrm{Te}$ & 15 & 450 & $3830.1(327.8)$ & $6407.2(286.5)$ & 3180.8 & Heating \\
\hline $\mathrm{Pb}_{0.96} \mathrm{Na}_{0.02} \mathrm{Eu}_{0.02} \mathrm{Te}$ & 15 & 500 & $2712.5(268.9)$ & $4125.7(228.5)$ & 2613.7 & Heating \\
\hline $\mathrm{Pb}_{0.96} \mathrm{Na}_{0.02} \mathrm{Eu}_{0.02} \mathrm{Te}$ & 15 & 550 & $2043.6(229.7)$ & 3408.7(196.6) & 2330.1 & Heating \\
\hline $\mathrm{Pb}_{0.96} \mathrm{Na}_{0.02} \mathrm{Eu}_{0.02} \mathrm{Te}$ & 15 & 600 & $1608.6(203.6)$ & 2662.7(170.6) & 2066.5 & Heating \\
\hline
\end{tabular}


Table 3 continued from previous page

\begin{tabular}{lllllll}
\hline Composition & Min Milled & $\mathrm{T}(\mathrm{K})$ & $\mathrm{S} 400$ & $\mathrm{~S} 220$ & $\mu$ strain & T Cycle \\
\hline $\mathrm{Pb}_{0.96} \mathrm{Na}_{0.02} \mathrm{Eu}_{0.02} \mathrm{Te}$ & 15 & 650 & $1278.0(189.7)$ & $1751.8(148.7)$ & 1757.2 & Heating \\
$\mathrm{Pb}_{0.96} \mathrm{Na}_{0.02} \mathrm{Eu}_{0.02} \mathrm{Te}$ & 15 & 700 & $968.1(170.8)$ & $1263.5(131.9)$ & 1513.1 & Heating \\
$\mathrm{Pb}_{0.96} \mathrm{Na}_{0.02} \mathrm{Eu}_{0.02} \mathrm{Te}$ & 15 & $666(25)$ & $887.4(428.8)$ & $1767.9(389.2)$ & 1624.3 & Cooling \\
$\mathrm{Pb}_{0.96} \mathrm{Na}_{0.02} \mathrm{Eu}_{0.02} \mathrm{Te}$ & 15 & $622(25)$ & $767.0(274.0)$ & $1427.9(245.2)$ & 1479.0 & Cooling \\
$\mathrm{Pb}_{0.96} \mathrm{Na}_{0.02} \mathrm{Eu}_{0.02} \mathrm{Te}$ & 15 & $576(25)$ & $1663.8(579.5)$ & $1666.8(412.9)$ & 1857.9 & Cooling \\
$\mathrm{Pb}_{0.96} \mathrm{Na}_{0.02} \mathrm{Eu}_{0.02} \mathrm{Te}$ & 15 & $522(25)$ & $959.8(302.4)$ & $1406.0(256.7)$ & 1540.5 & Cooling \\
$\mathrm{Pb}_{0.96} \mathrm{Na}_{0.02} \mathrm{Eu}_{0.02} \mathrm{Te}$ & 15 & $472(25)$ & $1030.8(240.3)$ & $1414.8(203.4)$ & 1567.3 & Cooling \\
$\mathrm{Pb}_{0.96} \mathrm{Na}_{0.02} \mathrm{Eu}_{0.02} \mathrm{Te}$ & 15 & $422(25)$ & $995.5(167.6)$ & $1226.9(138.3)$ & 1497.4 & Cooling \\
$\mathrm{Pb}_{0.96} \mathrm{Na}_{0.02} \mathrm{Eu}_{0.02} \mathrm{Te}$ & 15 & $372(25)$ & $985.6(132.4)$ & $1193.2(111.9)$ & 1481.1 & Cooling \\
\hline
\end{tabular}

\section{References}

1. Matsunaga, T. et al. Phase-change materials: Vibrational softening upon crystallization and its impact on thermal properties. Adv. Funct. Mater. 21, 2232-2239, DOI: 10.1002/adfm.201002274(2011).

2. Barron, T. H. K. \& Morrison, J. A. On the specific heat of solids at low temperatures. Can. J. Phys. 35, 799-810, DOI: 10.1139/p57-088 (1957).

3. Barron, T. H. K., Leadbetter, A. J., Morrison, J. A. \& Salter, L. S. The calculation of Debye-Waller factors from thermodynamic data. In Inelastic Scattering of Neutrons in Solids and Liquids, 49-57 (1962).

4. Barron, T. H. K., Leadbetter, A. J., Morrison, J. A. \& Salter, L. S. Temperature factors and thermodynamic properties of crystals. Acta Crystallogr. 20, 125-131, DOI: $10.1107 / \mathrm{s} 0365110 \times 66000227$ (1966).

5. Grimvall, G. Thermophysical Properties of Materials - Enlarged and revised edition. 89-97 (Elsevier Science B.V., 1999).

6. Hanus, R. et al. Lattice Softening Significantly Reduces Thermal Conductivity and Leads to High Thermoelectric Efficiency. Adv. Mater. 31, 1900108, DOI: 10.1002/adma.201900108 (2019).

7. Slade, T. J. et al. Contrasting SnTe-NaSbTe2and SnTe-NaBiTe2Thermoelectric Alloys: High Performance Facilitated by Increased Cation Vacancies and Lattice Softening. J. Am. Chem. Soc. 142, 12524-12535, DOI: 10.1021/jacs.0c05650 (2020).

8. Tan, G. et al. High Thermoelectric Performance in SnTe- AgSbTe 2 Alloys from Lattice Softening, Giant Phonon-Vacancy Scattering, and Valence Band Convergence. ACS Energy Lett. 3, 705-712, DOI: 10.1021/acsenergylett.8b00137 (2018).

9. Blackman, M. A Note on the Debye-Waller Theory. Acta Crystallogr. Sect. A 9, 734 (1956).

10. Vaccari, M. \& Fornasini, P. Einstein and Debye models for EXAFS parallel and perpendicular mean-square relative displacements. $J$. Synchrotron Radiat. 13, 321-325, DOI: 10.1107/S0909049506018504 (2006).

11. Wu, Y. et al. Lattice Strain Advances Thermoelectrics. Joule 3, 1276-1288, DOI: 10.1016/j.joule.2019.02.008(2019).

12. Wu, Y. et al. Manipulation of Band Degeneracy and Lattice Strain for Extraordinary PbTe Thermoelectrics. Research 2020, 1-12, DOI: $10.34133 / 2020 / 8151059$ (2020).

13. Houston, B., Strakna, R. E. \& Belson, H. S. Elastic constants, thermal expansion, and Debye temperature of lead telluride. J. Appl. Phys. 39, 3913-3916, DOI: 10.1063/1.1656874(1968).

14. Pei, Y. L. \& Liu, Y. Electrical and thermal transport properties of Pb-based chalcogenides: PbTe, PbSe, and PbS. J. Alloy. Compd. 514, 40-44, DOI: $10.1016 /$ j.jallcom.2011.10.036 (2012).

15. Nouneh, K. et al. Influence of an electron-phonon subsystem on specific heat and two-photon absorption of the semimagnetic semiconductors $\mathrm{Pb} 1-\mathrm{x} \mathrm{Ybx} \mathrm{X}(\mathrm{X}=\mathrm{S}, \mathrm{Se}, \mathrm{Te})$ near the semiconductor-isolator phase transformation. Phys. Rev. B - Condens. Matter Mater. Phys. 73, 4-11, DOI: 10.1103/PhysRevB.73.035329 (2006).

16. Matsushita, Y., Wianecki, P. A., Sommer, A. T., Geballe, T. H. \& Fisher, I. R. Type II superconducting parameters of Tl-doped PbTe determined from heat capacity and electronic transport measurements. Phys. Rev. B - Condens. Matter Mater. Phys. 74, 1-6, DOI: 10.1103/PhysRevB.74.134512 (2006).

17. Li, C. W. et al. Anharmonicity and atomic distribution of SnTe and PbTe thermoelectrics. Phys. Rev. B - Condens. Matter Mater. Phys. 90, 214303, DOI: 10.1103/PhysRevB.90.214303 (2014).

18. Bauer Pereira, P. et al. Lattice dynamics and structure of GeTe, SnTe and PbTe. Phys. Status Solidi (B) Basic Res. 250, 1300-1307, DOI: 10.1002/pssb.201248412 (2013).

19. Christensen, S., Bindzus, N., Sist, M., Takata, M. \& Iversen, B. B. Structural disorder, anisotropic micro-strain and cation vacancies in thermo-electric lead chalcogenides. Phys. Chem. Chem. Phys. 18, 15874-15883, DOI: $10.1039 / \mathrm{c} 6 \mathrm{cp01730d}$ (2016).

20. Božin, E. S. et al. Entropically stabilized local dipole formation in lead chalcogenides. Science 330, 1660-1663, DOI: 10.1126/science. 1192759 (2010).

21. Keiber, T., Bridges, F. \& Sales, B. C. Lead is not off center in PbTe: The importance of r-space phase information in extended x-ray absorption fine structure spectroscopy. Phys. Rev. Lett. 111, 1-5, DOI: 10.1103/PhysRevLett.111.095504 (2013).

22. Bridges, F., Keiber, T., Medling, S. \& Sales, B. C. Unusual distortion about Tl and Pb in PbTe:Tl. Phys. Stat. Sol. (C) 10, 236-241, DOI: 10.1002/pssc.201200478(2013). 